\title{
Considerations Regarding Input and Output Spaces for Time-Varying Dynamical Systems
}

\author{
William L. Root* \\ Aerospace Engineering Department, The University of Michigan \\ Communicated by A. V. Balakrishnan
}

\section{Introduction}

A small topic in the abstract theory of system modelling is investigated in this paper. As understood here, a system is a map $F$ from an input space $U$ to an output space $Y$. The systems with which we are concerned are causal dynamical systems for which inputs and outputs are functions of time. The topic under consideration is the choice and topologization of input and output spaces and their relation to system behavior. The concepts described below, which underlie the work in [1] and [2], provide intuitive background for the development here.

Let $y=F(u)$ be the input-output map of a causal (in general of a nonlinear, time-varying) system, where $u$ and $y$ are functions of time belonging to suitable function spaces $U$ and $Y$, respectively, and $F$ is causal, continuous and bounded. Let $P_{t}, t \in R$, denote projection on the past; i.e. for any function of time $z$, $\left(P_{t} z\right)(s)=z(s)$ or 0 according as $s \leqslant t$ or $s>t$. Fix $T>0$ and define $\tilde{F}_{t}$ for all $t \in R$ by

$$
\tilde{F}_{t}(u)=\left[P_{t}-P_{t-T}\right] F(u)
$$

where it is assumed that $U$ is a large enough space to contain all $P_{s} u, u \in U$. Each $\tilde{F}_{t}$ is the input-output map of a "truncated" system derived from $F$ for which the inputs are applied prior to $t$ and the outputs are observed over the interval $(t-T, t] . \tilde{F}_{t}$ is a map from the point-set $P_{t} U$ into the point-set $\left[P_{t}-P_{t-T}\right] Y$. If the spaces $U$ and $Y$ are invariant under arbitrary time-shifts, then elements of $P_{t} U$ may be identified with elements of $P_{0} U$, and elements of

* Research supported by Air Force Office of Scientific Research, Air Force Systems Command, USAF, under AFOSR Grant Number 72-2328. 
$\left[P_{t}-P_{t-T}\right] Y$ may be identified with elements of $\left[P_{0}-P_{-T}\right] Y$. Thus $\tilde{F}_{t}$ corresponds to a map $F_{t}: P_{0} U \rightarrow\left[P_{0}-P_{-T}\right] Y$. As $t$ varies, a trajectory of such maps is described in an appropriate space of maps from $P_{0} U$ into $\left[P_{0}-P_{-T}\right] Y$. This trajectory characterizes the time-varying behavior of the system; if $F$ is time-invariant the trajectory reduces to a single point.

One may consider a whole class of such systems and the corresponding class of trajectories. In [1] a mathematical framework is provided and a set of conditions stipulated such that under these conditions the trajectories of a class of causal systems are generated by a strongly continuous semigroup of linear transformations acting on a Banach space of mappings from $P_{0} U$ into $\left[P_{0}-P_{-T}\right] Y$. It is required that the maps $F_{t}$ have uniformly bounded finite memory. The input space $U$ is a metric subspace of any one of certain special Banach function spaces, and the output space $Y$ is also any one of these Banach function spaces. In the conference paper [2] the result just referred to in [1] is extended to classes of systems that no longer have finite memory. This extension is an easy modification of [1], but it requires that the truncated maps $F_{t}$ not be too sensitive to what happens in the indefinitely remote past. Since the $F_{t}$ are to be continuous, this in turn means that the topologization of the input space must be such as to become increasingly coarse as regards the behavior of inputs in the more and more distant past. Some form of this requirement seems to be essential, and part of the motivation for the present work was to investigate it more fully.

When the trajectories of a class of systems are generated by a strongly continuous semigroup of linear operators as described, the class has a linear, time-invariant structure even though the systems are individually nonlinear and time-varying-one may say the class of systems is "hyper-linear" and "hyper-time-invariant."

One would be pretty sure even without further study that the kind of system behavior described in [1] and [2] does not depend critically on the particular Banach spaces employed. So, partly as a matter of esthetics, but more to avoid having to paraphrase the proofs in [1] when similar results are wanted for other cases, a structure is developed here which is one level more abstract than that of [1] but yields corresponding results. In particular it includes the structures in [1] and [2] as special cases, and it is applicable to systems with stochastic outputs and stochastic inputs. (Stochastic systems as such are not discussed here, however.) This more abstract treatment brings out what appear to be essential features of the spaces and mappings involved, including especially the requirements on memory mentioned above.

We proceed as follows. In the next section families of normed linear function spaces parametrized by pairs of points on the real line are considered. Thus, $A_{s, t}$ will denote a normed linear function space whose elements are equivalence classes of functions on $R$, but for which two functions are necessarily equivalent if they agree on the interval $[s, t]$. If these spaces are interrelated in certain ways, they are called a fitted family of normed linear spaces. From the $A_{s, t}$, spaces $A_{t}=A_{-\infty, t}$ are derived. In certain cases the spaces $A_{t}$ are suitable as output spaces in mathematical models of input-output systems; special subsets of $A_{t}$ are suitable as input spaces. More precisely, the 
spaces $A_{t}$ can take the role of the moving observation spaces $\left[P_{t}-P_{t-T}\right] Y$ referred to above, and $U_{t} \subset A_{t}$ can take the role of $P_{t} U$. It is unnecessary to use the projections $P_{t}$.

Given a family $\left\{A_{s, t}\right\}$, a "bounding space" $A$ is introduced in such fashion that each element of $A$ corresponds to an element of each $A_{s, t}$ (although of course not vice versa). The global system will be modelled as a mapping $F$ from $U$, a subset of $A$, into $Y$ where $Y$ is a bounding space for some (possibly different) fitted family of normed spaces. We shall use the notations $\left\{A_{s, t}\right\},\left\{A_{t}\right\}$ and $A$ for spaces related to inputs, and $\left\{B_{s, t}\right\},\left\{B_{t}\right\}$, and $B$ for output spaces. Thus $Y=B$, and $F: U \rightarrow B$. Conditions will be imposed so that, as above, $F$ induces a map $\tilde{F}_{t}: U_{t} \rightarrow B_{t}$, and again this will correspond to a map $F_{t}: U_{0} \rightarrow B_{0}$. Truncated-system trajectories are then defined as above.

In the last section it is shown that with certain reasonable properties assumed for the input and output spaces and for $F$ the trajectories are continuous. Conditions are then established for the hyper-linearity and hyper-time-invariance mentioned above.

In order to have a satisfactory mathematical system model there are requirements which input and output spaces and input-output maps should meet, depending of course on circumstances. Considering that we are concerned with causal systems with well-defined inputs and outputs, which are to operate for indefinite periods of time and which are in general nonlinear and time-varying, there are certain general conditions which seem desirable. These will be postulated below. One of the objectives of this work is to investigate the properties of fitted families of normed linear spaces in order to establish hypotheses under which these conditions are met. A second objective is to show that when these conditions are met a truncated-system trajectory theory makes sense.

The conditions we set are:

(1) input spaces $U$ and output spaces $Y$ should be shift-invariant; i.e. if $L_{s}$ denotes translation to the left by $s, L_{s}(U)=U, L_{s}(Y)=Y, s \in R$.

(2) The spaces $U_{t}$ and $Y_{t}$ (whose elements represent, respectively, inputs and outputs over all or some portion of the past up to the present) should be shift-continuous; i.e. $L_{h} u \rightarrow u$ in the metric of $U_{t}$ as $h \rightarrow 0$ and $L_{h} y \rightarrow y$ in the metric of $Y_{t}$ as $h \rightarrow 0$.

(3) Within the overlying structure (i.e. with a given family $\left\{A_{s, t}\right\}$ ) it should be possible to choose input spaces $U$ which satisfy the other criteria and are also such that each $U_{t}$ is totally bounded, while at the same time, of course, being "large enough" sets to contain the inputs of interest.

(4) The maps $F_{t}$ should be continuous and bounded.

Not much argument will be offered here to justify these requirements (there is quite a bit of related discussion in [1]). Condition (2) is relevant to the continuity of truncated-system trajectories. (3) can be important in modelling nonlinear systems, where restrictions on the class of inputs are usually important. (4) is more or less arbitrary; some regularity condition on the input-output maps is needed and we have chosen a mild one. 


\section{Fitted Families of Normed Linear Spaces}

Let $C$ be a linear space of functions from $R$ into a fixed real Banach space $\mathscr{B}$ such that any translate of a function in $C$ is also a function in $C$. Let $N=\left\{\|\cdot\|_{s, t},-\infty<s<t<\infty\right\}$ be a family of seminorms on $C$ satisfying the following conditions:

1. If $f_{1}, f_{2} \in C$ and $f_{1}(\tau)=f_{2}(\tau), s \leqslant \tau \leqslant t$, then $\left\|f_{1}-f_{2}\right\|_{s, t}=0$.

2. $\left\|L_{\tau} f\right\|_{s-\tau, t-\tau}=\|f\|_{s, t}, f \in C,-\infty<\tau<\infty$.

3. If $r \leqslant s \leqslant t,\|f\|_{s, t} \leqslant\|f\|_{r, t}, f \in C$.

4. If $r \leqslant s \leqslant t,\|f\|_{r, t} \leqslant\|f\|_{r, s}+\|f\|_{s, t}, f \in C$.

5. For some $\alpha>0(\alpha$ may be $+\infty)$ and $K \geqslant 1$, and for all $f \in C$, $\|f\|_{r, s} \leqslant K\|f\|_{r, t}, r \leqslant s \leqslant t$, whenever $t-r<\boldsymbol{\alpha}$.

A pair $(C, N)$ 'satisfying these conditions is called a fitted family of seminorms on $C$. The normed linear space formed from equivalence classes of functions in $C$ with norm $\|\cdot\|_{s, t}$ is denoted $A_{s, t}$. The elements of $A_{s, t}$ are the equivalence classes of functions from $C$ given by: $f \sim g$ iff $\|f-g\|_{s, t}=0$. The set $\left\{A_{s, t}\right\}$ is the fitted family of normed linear spaces given by $(C, N)$. It is to be observed that the equivalence classes for different $(s, t)$ pairs may or may not be simply related to each other. For example, if $r<s<t,\left\|f_{1}-f_{2}\right\|_{r, t}=0$ implies $\left\|f_{1}-f_{2}\right\|_{s, t}=0$, but does not imply $\left\|f_{1}-f_{2}\right\|_{r, s}=0$ in general. The difficulty is caused by the rather weak condition (5) which stands in place of the stronger requirement, $\|f\|_{r, s} \leqslant\|f\|_{r, t}, r \leqslant s \leqslant t$. Unfortunately this latter condition simply does not hold in many situations we wish to include.

\section{Examples}

(A) $C$ is the set of strongly measurable (Lebesgue) functions $f$ from $R$ to $\mathscr{B}$ such that

$$
\|f\|_{s, t}=\left(\int_{s}^{t}|f(v)|^{p} d v\right)^{1 / p}, \quad 1 \leqslant p<\infty
$$

exists for each pair $(s, t),-\infty<s \leqslant t<\infty . N$ is the set of $L_{p}$ seminorms defined on $C$ by Eq. (1). In this example, condition (5) is satisfied in its trivial form, of course, with $\alpha=\infty, K=1$.

(B) Example (A) is modified as follows. Fix $T>0$. If $t-s \leqslant T$, define $\|f\|_{s, t}$ as above; if $t-s>T$, define $\|f\|_{s, t}$ by

$$
\|f\|_{s, t}=\left(\int_{t-T}^{t}|f(v)|^{p} d v\right)^{1 / p}, \quad 1 \leqslant p<\infty .
$$

Condition (5) is satisfied with $\alpha=T, K=1$, but for no larger $\alpha$.

(C) Example (A) is modified by using a weighting function. In particular let $\phi: R \rightarrow R$ be a function satisfying: $\phi(t)=0$ for $t<0 ; \phi(0)=1 ; \phi$ monotone nonincreasing on $[0, \infty) ; \phi(t) \rightarrow 0$ as $t \rightarrow \infty$, and $\int_{0}^{\infty} \phi(t) d t=A, 0<A<\infty$. Then 
put

$$
\|f\|_{s, t}=\left(\int_{s}^{t}|f(v)|^{p} \phi(t-v) d v\right)^{1 / p}
$$

This example contains (B) as a special case.

(D) Example (C) is modified as follows.

$$
\|f\|_{s, t}=\sup _{s<t^{\prime}<t}\left(\int_{s}^{t^{\prime}}|f(v)|^{p} \phi\left(t^{\prime}-v\right) d v\right)^{1 / p}
$$

(E) $C$ is the class of continuously differentiable functions from $R$ to $R$

$$
\|f\|_{s, t}=\max _{s \leqslant \tau \leqslant t}\left|f^{\prime}(\tau)\right|
$$

(F) Example (E) is modified by using a weighting function $\phi$ as described in (C), except that the integrability condition is not required.

$$
\|f\|_{s, t}=\max _{s \leqslant \tau \leqslant t}\left|f^{\prime}(\tau) \phi(t-\tau)\right|
$$

Some of these examples will be expanded upon later.

We note that if $\left(C^{\prime}, N^{\prime}\right)$ and $\left(C^{\prime \prime}, N^{\prime \prime}\right)$ are each fitted families of seminorms, then $(C, N)$ is also, where $C=C^{\prime} \cap C^{\prime \prime}$ and the norms $\|\cdot\|_{s, t}$ in $N$ are given by

$$
\|f\|_{s, t}=a\|f\|_{s, t}^{\prime}+b\|f\|_{s, t}^{\prime \prime}
$$

with $a, b \geqslant 0$, and $\|\cdot\|_{s, t}^{\prime} \in N^{\prime},\|\cdot\|_{s, t}^{\prime \prime} \in N^{\prime \prime}$. Also, from the shift-invariance and condition (2) it follows that any $A_{s, t}$ is isomorphic to $A_{0, t-s}$ under the shift $L_{s}$. Consequently the family $N$ of seminorms can be defined in terms of a single-parameter family, $\left\{\|\cdot\|_{0, r}\right\}, 0 \leqslant r<\infty$.

We extend the class of seminorms so as to be able to account for the behavior of functions on the entire left half-line. Let $C_{0}=\left\{f \in C: \lim _{s \rightarrow-\infty}\|f\|_{s, t}\right.$ exists, $t \in R\}$. For $f \in C_{0}$, define $\|f\|_{-\infty, t}=\lim _{s \rightarrow-\infty}\|f\|_{s, t}$; obviously, $\|\cdot\|_{-\infty, t}$ is a seminorm on $C_{0}$. Henceforth $\|f\|_{-\infty, t}$ will usually be denoted $\|f\|_{t}$. We now put $\bar{N}=\left\{\|\cdot\|_{s, t},-\infty \leqslant s<t<\infty\right\}$, where $\|f\|_{-\infty, t}$ is defined only for $f \in C_{0} \subset C$. It is clear that the properties (1),..,(4) still hold for $\left(C_{0}, \bar{N}\right)$ if the left-most point is replaced by $-\infty$; (5) holds for $r=-\infty$ if $\alpha=\infty$, otherwise the property is irrelevant to the case $r=-\infty$. The normed linear space formed from equivalence classes of functions in $C_{0}$ with norm $\|\cdot\|_{t}$ is denoted $A_{t} \cdot C_{0}$ is also invariant under shifts, so, in analogy to a remark made above, it follows that any $A_{t}$ is isomorphic to $A_{0}$ under a shift operation.

Given $(C, N)$, it is useful to define another, related, fitted family of seminorms on $C$ and the corresponding fitted family of normed linear spaces. First, define $\|\cdot\|^{s, t}$ by

$$
\|f\|^{s, t}=\sup _{s \leqslant \tau \leqslant t}\|f\|_{s, \tau}, \quad f \in C .
$$


Clearly $\|\cdot\|^{s, t}$ is a seminorm. There is a question as to whether $\|f\|^{s, t}$ is finite for all $f \in C$ and all pairs $(s, t),-\infty<s \leqslant t<\infty$, and a second question as to whether the families are fitted. Routine verifications show the answer to be yes to both questions; we carry out a couple of these below. Put $M=$ $\left\{\|\cdot\|^{s, t},-\infty<s \leqslant t<\infty\right\}$.

Proposition 1. $(C, M)$ is a fitted family of seminorms on $C$.

Proof. For arbitrary $(s, t),-\infty<s \leqslant t<\infty$, and any $f \in C,\|f\|^{s, t}$ exists. For suppose the contrary, then there is a sequence $\left\{\tau_{k}\right\}$ such that $\|f\|_{\mathrm{s}, \tau_{k}} \geqslant k$, $k=1,2, \ldots$. By compactness, we may suppose the $\tau_{k}$-sequence chosen so that $\tau_{k} \rightarrow \tau$ as $k \rightarrow \infty$. Let $0<\beta<\alpha / 2$ and consider $\tau_{k}$ such that $\left|\tau_{k}-\tau\right| \leqslant \beta$. Then by conditions (4), (5)

$$
\|f\|_{s, \tau_{k}} \leqslant\|f\|_{s, \tau-\beta}+\|f\|_{\tau-\beta, \tau_{k}} \leqslant\|f\|_{s, \tau-\beta}+K\|f\|_{\tau-\beta, \tau+\beta}
$$

But the right side is a fixed number, so there is a contradiction.

To show $(C, M)$ is fitted it is necessary to verify the fitted-family properties (1),..,(5). Consider (4), for example. Given $\epsilon>0$ there is $\tau, r \leqslant \tau \leqslant t$, such that

$$
\|f\|^{r, t} \leqslant\|f\|_{r, \tau}+\epsilon .
$$

Consider the two possible cases: $\tau \geqslant s, \tau<s$. If $\tau \geqslant s$, then

$$
\begin{aligned}
\|f\|_{r, \tau}+\epsilon & \leqslant\|f\|_{r, s}+\|f\|_{s, \tau}+\epsilon \leqslant\|f\|^{r, s}+\sup _{s \leqslant \tau^{\prime} \leqslant \tau}\|f\|_{s, \tau^{\prime}}+\epsilon \\
& \leqslant\|f\|^{r, s}+\sup _{s \leqslant \tau^{\prime} \leqslant t}\|f\|_{s, \tau^{\prime}}+\epsilon=\|f\|^{r, s}+\|f\|^{s, t}+\epsilon .
\end{aligned}
$$

If $\tau<s$, then

$$
\|f\|_{r, \tau}+\epsilon \leqslant \sup _{r \leqslant \tau^{\prime} \leqslant s}\|f\|_{r, \tau^{\prime}}+\epsilon=\|f\|^{r, s}+\epsilon .
$$

Since $\epsilon$ is arbitrary, one has in either case that $\|f\|^{r, t} \leqslant\|f\|^{r, s}+\|f\|^{s, t}$.

The other conditions can be established by equally simple arguments.

Remark 1. Condition (5) is satisfied by the family $M$ with $\alpha=\infty, K=1$.

From Proposition 1 it follows that $(C, M)$ determine a fitted family of normed linear spaces, which will be denoted $\left\{A^{s, t}\right\}$. Then for a class of functions $C^{0} \subset C, M$ can be extended exactly as above to a class $\bar{M}=\left\{\|\cdot\|^{s, t},-\infty \leqslant s<t<\infty\right\}$, where $\|f\|^{-\infty, t}$ is defined only for $f \in C^{0}$.

Remark 2. It is easily seen that

$$
\|f\|^{-\infty, t}=\lim _{s \rightarrow-\infty}\|f\|^{s, t}=\sup _{-\infty<\tau \leqslant t}\|f\|_{-\infty, \tau} .
$$

As before, we write $\|f\|^{-\infty, t}=\|f\|^{t}$.

The class of functions $C^{0}$ is given by $C^{0}=\left\{f \in C: \lim _{s \rightarrow-\infty}\|f\|^{s, 0}\right.$ exists $\}$. Since both $\|f\|^{s, 0}$ and $\|f\|_{s, 0}$ are monotone nondecreasing as $s \rightarrow-\infty$, and since 
$\|f\|^{s, 0} \geqslant\|f\|_{s, 0}$ it follows that $C^{0} \subset C_{0}$. They may or may not be equal. If $(C, N)$ is such that $M=N,(C, N)$ is said to be irregular; otherwise $(C, N)$ is regular. Obviously if $(C, N)$ is irregular, $C^{0}=C_{0}$. Also, any $(C, M)$ is irregular. Of the examples listed, (A), (D) and (E) are irregular. If the family of norms in example (C) is denoted $N$, then the family of norms in (D) is the corresponding $M$.

One more space determined by $(C, N)$ is to be introduced. Put

$$
\|f\|=\sup _{t \in R}\|f\|_{t}
$$

for all $f \in C_{0}$ for which the right side exists. The linear space of all such $f$ is denoted $C_{00}$, and the normed linear space of equivalence classes of $C_{00}$ with respect to this norm is denoted $A . A$ is said to be the bounding space for the family $\left\{A_{s, t}\right\}$. We shall justify this term, but first some elementary facts about the norm $\|\cdot\|$ are noted.

\section{Lemma 1}

$$
\|f\|=\sup _{(s, t), s \leqslant t}\|f\|_{s, t}
$$

$$
\|f\|=\sup _{t}\|f\|^{t}
$$

$$
\begin{aligned}
& \text { (3) }\|f\|=\sup _{(s, t), s \leqslant t}\|f\|^{s, t} \\
& \text { (4) }\|f\|=\left\|L_{\tau} f\right\|, \tau \in R
\end{aligned}
$$

Proof. The proofs are all easy verifications based directly on the definitions. In (1) and (3) the supremum is over all pairs $(s, t)$ with $s \leqslant t$.

It follows from Lemma 1 that $C_{00}$ is a shift-invariant linear subspace of $C^{0}$ as well as of $C_{0}$, and that $A$ is the bounding space for the family $\left\{A^{s, t}\right\}$ as well as for the family $\left\{A_{s, r}\right\}$.

The connection between the bounding space $A$ and other spaces considered can be stated in terms of a partial ordering. Let $A_{1}, A_{2}$ be the normed linear spaces formed from $\left(C_{1},\|\cdot\|_{1}\right)$ and $\left(C_{2},\|\cdot\|_{2}\right)$, respectively. $C_{1}$ and $C_{2}$ are each to be shift-invariant linear subspaces of $C$ with $C_{1} \supset C_{2} ;\|\cdot\|_{1}$ and $\|\cdot\|_{2}$ are seminorms defined on $C_{1}$ and $C_{2}$, respectively (the elements of $A_{i}$ are then equivalence classes of functions with respect to $\|\cdot\|_{i}$ ), with $\|f\|_{1} \leqslant\|f\|_{2}$ for $f \in C_{2}$. This defines a partial ordering, and we write $A_{1}<A_{2}$. It follows from Lemma 1 that $A_{s, t}<A, A_{t}<A, A^{s, t}<A$ and $A^{t}<A$. Furthermore:

Lemma 2. $A$ is the least upper bound (1.u.b.) of each of the following families of normed linear spaces: $\left\{A_{s, t}\right\},\left\{A_{t}\right\},\left\{A^{s, t}\right\}\left\{A^{t}\right\}$.

Proof. We prove the case, $A=$ l.u.b. $\left\{A_{s, t}\right\}$; the others are similar. Suppose $A^{\prime}$ formed from $\left(C^{\prime},\|\cdot\|^{\prime}\right)$ satisfies $A^{\prime}>A_{s, t}$ for each $(s, t)$ pair. Then, for $f \in C^{\prime}$,

$$
\|f\|_{s, t} \leqslant\|f\|^{\prime} \text { for all }-\infty<s \leqslant t<\infty .
$$

Hence $f \in C_{00}$, so that $C^{\prime} \subset C_{00}$. Also,

$$
\|f\|=\sup _{(s, t)}\|f\|_{s, t} \leqslant\left\|f^{\prime}\right\| .
$$


That is, $A<A^{\prime}$.

Example ( $\left.\mathbf{B}^{\prime}\right)$. Let $A_{s, t}$ be as given by Example (B). Then the bounding space $A$ is the space $\mathcal{L}_{T}^{(p)}$ introduced in [1]. It is shown there to be a Banach space (actually in [1] the functions making up ${ }_{-}^{(p)}$ were assumed to have values in $R^{n}$, rather than $\mathscr{B}$ ).

Under certain general conditions the spaces $A_{t}$ are suitable for use as input spaces for causal time systems according to the criteria given in the Introduction; under certain other conditions they are not suitable. At this point two definitions are needed in order to set up these conditions.

Definition. $(C, N)$ has the support property (property (S)) if for any $(s, t)$, $-\infty<s<t<\infty$ there is a function $f \in C$ which satisfies $\|f\|_{s, t} \neq 0$ and $f(\tau)=0$ for $\tau<s, \tau>t$.

Definition. Consider a family $\left\{A_{s, t}\right\}$. For any $c>0$ and any $t$ let

$$
G(c, t)=\left\{f \in C_{0}:\|f\|_{\tau} \leqslant c, \quad \forall \tau \leqslant t\right\} .
$$

Then $\left\{A_{s, t}\right\}$ is tapered if for any $\epsilon>0, c>0$ and $t \in R$, there is a positive number $\delta=\delta(\epsilon, c, t)$ such that

$$
\|f\|_{t} \leqslant\|f\|_{t-\delta, t}+\epsilon
$$

for all $f \in G(c, t)$.

Remark 3. Since $G(c, s)=L_{t-s}[G(c, t)]$ it follows that $\delta$ does not in fact depend on $t$. For $s<t$, it is also true that $G(c, t) \subset G(c, s)$, hence the conclusion above can be strengthened to read: there is a positive number $\delta=\delta(\epsilon, c)$ such that

$$
\|f\|_{s} \leqslant\|f\|_{s-\delta, s}+\epsilon
$$

for all $f \in G(c, t)$ and any $s \leqslant t$.

Example (C). We show that $\left\{\boldsymbol{A}_{s, t}\right\}$ is tapered. From the conditions on $\phi$ one has: for some $T>0$ there is $a>0$ such that $\phi(t) \geqslant a, 0 \leqslant t<T$. Then, for any $f \in G(c, t)$,

$$
\begin{aligned}
a \int_{\tau-T}^{\tau}|f(v)|^{p} d v & \leqslant \int_{\tau-T}^{\tau}|f(v)|^{p} \phi(\tau-v) d v \\
& \leqslant \int_{-\infty}^{\tau}|f(v)|^{p} \phi(\tau-v) d v<c^{p}, \tau \leqslant t
\end{aligned}
$$

or,

$$
\int_{\tau-T}^{\tau}|f(v)|^{p} d v \leqslant \frac{c^{p}}{a}, \tau \leqslant t
$$


Then, for any $n=1,2, \ldots$,

$$
\|f\|_{t}^{p}=\|f\|_{t-n T, t}^{p}+\int_{-\infty}^{t-n T}|f(v)|^{p} \phi(t-v) d v
$$

The integral can be written as

$$
\sum_{k=n}^{\infty} \int_{t-(k+1) T}^{t-k T}|f(v)|^{p} \phi(t-v) d v \leqslant \frac{c^{p}}{a} \sum_{k=n}^{\infty} \phi(k T)
$$

Now, since $\int_{0}^{\infty} \phi(s) d s=A<\infty$ and since $\phi$ is nonincreasing, $\sum_{k=n}^{\infty} \phi(k T) \rightarrow 0$ as $n \rightarrow \infty$. Thus for sufficiently large $n_{0}$, the integral term does not exceed $\epsilon^{p}$ for any $n \geqslant n_{0}$ for all $f \in G(c, t)$, and

$$
\|f\|_{t}^{p} \leqslant\|f\|_{t-n T, t}^{p}+\epsilon^{p} \leqslant\left(\|f\|_{t-n T, t}+\epsilon\right)^{p}, n \geqslant n_{0} .
$$

Note that this also shows that the family $\left\{A_{s, t}\right\}$ of Example (B) is tapered. The spaces of Example (C) are those used in [2] with $p=2\left(A_{t}=L_{2}\left(\mu_{t}\right)\right.$ in the notation of that paper). Thus, tapered input spaces are used in [2], although the abstract concept is not introduced there.

By an easy argument using calculations similar to those above one sees that the bounding spaces for Examples (B) and (C) have equivalent norms, no matter what $\phi$ may be as long as it satisfies the weighting-function conditions (see [2], Lemma 2.). It is shown in [1] that the bounding space for Example (B) is a Banach space, so the bounding space for Example (C) with arbitrary $\phi$ is also a Banach space.

Proposition 2. If $\left\{A_{s, t}\right\}$ has property $(S)$, then $\left\{A^{t, s}\right\}$ is not tapered.

Proof. Let $f \in C, f(\tau)=0$ for $\tau>-1$ or $\tau \geqslant 0$, and $\|f\|_{0}=\|f\|_{-1,0}>0$. Then $\|f\|^{0}=\mathrm{c} \geqslant\|f\|_{0}>0$. For any $b>1,\left\|L_{b} f\right\|^{0}=\left\|L_{b} f\right\|^{-b}=c$. In fact, $\left\|L_{b} f\right\|^{-b}=\|f\|^{0}$ by condition (2). But $\left\|L_{b} f\right\|^{0} \leqslant\left\|L_{b} f\right\|^{-b}+\left\|L_{b} f\right\|^{-b, 0}=\left\|L_{b} f\right\|^{-b}$ since $L_{b} f$ vanishes on the interval $[-b, 0]$; and $\left\|L_{b} f\right\|^{-b} \leqslant\left\|L_{b} f\right\|^{0}$ by condition (5) for the family $\left\{A^{s, t}\right\}$.

Now suppose $\left\{A^{s, t}\right\}$ is tapered. Then, given $\epsilon>0$ there is $\delta=\delta(c, \epsilon)$ such that $\left\|L_{b} f\right\|^{0}-\left\|L_{b} f\right\|^{-\delta, 0} \leqslant \epsilon$. Take $\epsilon=c / 2$ and $b>1+\delta$, then $\left\|L_{b} f\right\|^{0}-\left\|L_{b} f\right\|^{-\delta, 0}=$ $c-0>c / 2$ which is a contradiction.

The bound provided by the following lemma is useful in discussing shift continuity.

Lemma 3. For any $(C, N)$, any $f \in C_{0}$, and any $\tau$ satisfying $|\tau| \leqslant \alpha$,

$$
\left\|L_{\tau} f-f\right\|_{t} \leqslant\|f\|_{t-\alpha}+K\|f\|_{t-\alpha, t}+2\|f\|_{t}+K\|f\|_{t, t+\alpha}
$$

where $(\alpha, K)$ are as in condition (5). 
Proof. Take $0 \leqslant \tau \leqslant \alpha$, then

$$
\begin{aligned}
\left\|L_{\tau} f-f\right\|_{t} & \leqslant\left\|L_{\tau} f\right\|_{t}+\|f\|_{t}=\|f\|_{t+\tau}+\|f\|_{t} \\
& \leqslant 2\|f\|_{t}+\|f\|_{t, t+\tau} \leqslant 2\|f\|_{t}+K\|f\|_{t, t+\alpha}
\end{aligned}
$$

and

$$
\begin{aligned}
\left\|L_{-\tau} f-f\right\|_{t} & \leqslant\left\|L_{-\tau} f\right\|_{t}+\|f\|_{t}=\left\|f_{t-\tau}\right\|+\|f\|_{t} \\
& \leqslant\|f\|_{t-\alpha}+\|f\|_{t-\alpha, t-\tau}+\|f\|_{t} \leqslant\|f\|_{t-\alpha}+K\|f\|_{t-\alpha, t}+\|f\|_{t} .
\end{aligned}
$$

Proposition 3. If $\left\{A_{s, t}\right\}$ is shift-continuous and tapered then $\left\|L_{\tau} f-f\right\|_{t} \rightarrow 0$ as $\tau \rightarrow 0$ for all $f \in C^{0}\left(\subset C_{0}\right)$.

Proof. It follows immediately from the preceding Lemma that with $|\tau| \leqslant \alpha$,

$$
\left\|L_{\tau} f-f\right\|_{t} \leqslant\|f\|_{t-\alpha}+(K+1)\|f\|_{t}+K\|f\|_{t+\alpha} .
$$

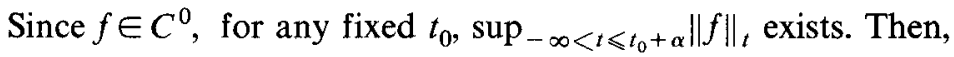

$$
\left\|L_{\tau} f-f\right\|_{t} \leqslant \text { constant }=a, \text { for } t \leqslant t_{0}
$$

so that the functions $\left(L_{\tau} f-f\right)$ all belong to $G\left(a, t_{0}\right)$ for $|\tau| \leqslant \alpha$. Then, for $\epsilon>0$ there is $\delta>0$ such that

$$
\left\|L_{\tau} f-f\right\|_{t}-\left\|L_{\tau} f-f\right\|_{t-\delta, t} \leqslant \epsilon, \quad t \leqslant t_{0} .
$$

Since $\left\{A_{s, t}\right\}$ is shift-continuous and $t_{0}$ is arbitrary, the conclusion follows.

Proposition 3 does not cover the standard cases of $L_{p}$ spaces, since they are not tapered. For the $L_{p}$-spaces, $1 \leqslant p<\infty$, the fact that the condition $\|f\|_{r, t}^{p}=\|f\|_{r, s}^{p}+\|f\|_{s, t}^{p}$ holds instead of just the weaker condition (4) is of course sufficient for the proof, without regard to the tapering property. As will be seen, $L_{p}$ spaces on $R$ or on the half-line are of little interest here in any event.

To set off against the preceding Proposition we have the following:

Proposition 4. If $\left\{A_{s, t}\right\}$ has property $(S)$ and is tapered, and if $A_{0}$ (and hence each $\left.A_{t}\right)$ is a Banach space, then there is a function $f \in C^{0}$ such that $\left\|L_{\tau} f-f\right\|^{0}$ does not approach zero as $\tau \rightarrow 0$. That is, $A^{t}$ is not shift-continuous.

\section{Proof. See Appendix.}

The proof consists of constructing a function $f$ consisting of an infinite string of isolated "bumps" which get narrower and taller as $t \rightarrow-\infty$. It is necessary that $A_{0}$ be complete in order to make this construction.

We now consider the existence of totally bounded shift-invariant subsets of $A_{t}$. It is irrelevant to this discussion whether the spaces $A_{s, t}, A_{t}$ are complete or not. It is convenient to use the following alternative characterization of total boundedness: a subset $S$ of a metric space is totally bounded if every sequence of points in $S$ has a Cauchy subsequence. 
Lemma 4. Let $T>0$ be arbitrary, and $S$ be a subset of $C$. If $L_{t} S=\left\{L_{t} f: f \in S\right\}$ is totally bounded in $A_{-T, 0}$ for each $t \in R$, then $S$ is totally bounded in every $A_{s, t}$.

Proof. The hypothesis is equivalent to: $S$ is totally bounded in every $A_{t-T, t}$. First, suppose $t-s<T$, then if $\left\{f_{n}\right\}$ is Cauchy in $A_{t-T, t}$ it is also Cauchy in $A_{s, t}$ by condition (3). Thus $S$ is totally bounded in any $A_{s, t}$ with $t-s<T$.

On the other hand, if $\left\{f_{n}\right\}$ is a sequence of points in $S$ which is Cauchy in $A_{t-T, t}$, there is a subsequence $\left\{f_{1 n}\right\}$ which is Cauchy on $A_{t-2 T, t-T}$, by the hypothesis on $S$. By condition (4), $\left\{f_{1 n}\right\}$ is also Cauchy in $A_{t-2 T, t}$, so $S$ is totally bounded in $A_{t-2 T, t}$. By induction, $S$ is totally bounded on any $A_{s, t}$ for which $t-s=k T, k=1, \ldots$. Then, as in the first remark, $S$ is totally bounded in any $A_{s, t},-\infty<s<t<\infty$.

Lemma 5. Let $S \subset C$ be totally bounded in each $A_{s, t}$ and in addition be uniformly bounded over all $A_{s, t}$. If $\left\{A_{s, t}\right\}$ is tapered, then $S$ is totally bounded in each $A_{t}$.

Proof. By the uniform bound hypothesis, $\|f\|_{t}$ exists and does not exceed some constant $\gamma$, for all $f \in S$ and all $t$. Consider $G(2 \gamma, t)$ for an arbitrarily fixed $t$; given $\epsilon>0$ there is $\delta>0$ such that

$$
\|g\|_{t} \leqslant \epsilon+\|g\|_{t-\delta, t} \quad \text { for all } g \in G(2 \gamma, t) .
$$

From any given sequence of points in $S$ extract a subsequence $\left\{f_{n}\right\}$ which is Cauchy in $A_{t-\delta, t}$ and let $m, n$ be large enough that $\left\|f_{n}-f_{m}\right\|_{t-\delta, t} \leqslant \epsilon$. Then, since $\left(f_{n}-f_{m}\right) \in G(2 \gamma, t)$,

$$
\left\|f_{n}-f_{m}\right\|_{t} \leqslant \epsilon+\left\|f_{n}-f_{m}\right\|_{t-\delta, t} \leqslant 2 \epsilon .
$$

The following Lemma is used here and also in the next section.

Lemma 6. Let $\beta$ satisfy $0<\beta \leqslant \alpha / 2$; let $-\infty \leqslant a<b<\infty$, and let $U$ be a subset of $C$ that satisfies:

(i) $\left\|L_{\tau} f-f\right\|_{a, b} \rightarrow 0$ as $\tau \rightarrow 0, f \in U$.

(ii) $U$ is totally bounded in $A_{a-\beta, b-\beta}$ and also in $A_{b-\beta, b+\beta}$.

Then $U$ is uniformly shift continuous in $A_{a, b}$; i.e., (i) is satisfied uniformly.

Proof. It follows readily from conditions (3), (4), (5) that for any $\tau,|\tau| \leqslant \beta$, and with $\beta$ as specified, $0<\beta \leqslant \alpha / 2$,

$$
\|f\|_{a-\tau, b-\tau} \leqslant\|f\|_{a-\beta, b-\beta}+K\|f\|_{b-\beta, b+\beta} .
$$

For all $f, g \in U$ we define

$$
d(f, g)=\|f-g\|_{a-\beta, b-\beta}+K\|f-g\|_{b-\beta, b+\beta} .
$$

$d(\cdot, \cdot)$ is a metric on equivalence classes of functions in $U$; call the metric space so defined, $\tilde{U}$. From hypothesis (ii) it is easily seen that $\tilde{U}$ is totally bounded. 
Hence, for any $\epsilon>0$, there is an $\epsilon$-net of points $w_{i}, i=1, \ldots, N(\epsilon)$, for $\tilde{U}$.

We now have

$$
\begin{aligned}
\left\|L_{\tau} f-f\right\|_{a, b} & \leqslant\left\|L_{\tau} f-L_{\tau} w_{i}\right\|_{a, b}+\left\|L_{\tau} w_{i}-w_{i}\right\|_{a, b}+\left\|w_{i}-f\right\|_{a, b} \\
& \leqslant\left\|f-w_{i}\right\|_{a-\tau, b-\tau}+\left\|L_{\tau} w_{i}-w_{i}\right\|_{a, b}+\left\|w_{i}-f\right\|_{a, b}
\end{aligned}
$$

for any $w_{i}$. Choose $\tau$ small enough that $\left\|L_{\tau} w_{i}-w_{i}\right\|_{a, b} \leqslant \epsilon$ for each $w_{i}, i=1, \ldots, N$, and also that $|\tau| \leqslant \beta$. Then for any $f \in U$ there is a $w_{i}$ such that

$$
\left\|f-w_{i}\right\|_{a-\tau, b-\tau} \leqslant d\left(f, w_{i}\right) \leqslant \epsilon
$$

and

$$
\left\|f-w_{i}\right\|_{a, b} \leqslant d\left(f, w_{i}\right) \leqslant \epsilon,
$$

so that

$$
\left\|L_{\tau} f-f\right\|_{a, b} \leqslant 3 \epsilon .
$$

Note that the proof is valid for $a=-\infty$ as well as for $a>-\infty$.

Proposition 5. For fixed $a, b,-\infty<a<b<\infty$, let $M_{0} \subset C$ be a set of functions that vanish outside the interval $(a, b)$. Further let it be required that $M_{0}$ is totally bounded in every $A_{s, t}$ with $a \leqslant s \leqslant t \leqslant b$. Then if $\left\{A_{s, t}\right\}$ is tapered and the $A_{s, t}$ are shift-continuous, the shift-invariant set $M=\left\{L_{\tau} f: f \in M_{0}, \tau \in R\right\}$ is totally bounded and shift-continuous in each $A_{t}$. If $C$ has property $(S)$ a nontrivial $M_{0}$ satisfying the conditions is guaranteed to exist.

Proof. There is no loss in generality in assuming $a=0$, so that the functions in $M_{0}$ are supported in $[0, b]$. We show first there is $\gamma>0$ such that $\|f\|_{s, t} \leqslant \gamma$ for all $f \in M_{0}$ and all $s, t,-\infty<s \leqslant t<\infty$. Suppose to start with that $b>\alpha$ and partition the interval $[0, b]$ at the points $(0, \alpha, 2 \alpha, \ldots, k \alpha, b)$, where $\alpha \geqslant b-k \alpha>0$. Since $M_{0}$ is totally bounded in each of the $A_{i \alpha, j \alpha}$ and in $A_{i \alpha, b}$, where $i<j$ are integers between 0 and $k$, there is a constant $\gamma^{\prime}$ such that the norm of $f$ in each of these spaces is less then $\gamma^{\prime}$. Then by conditions (3), (4), (5) it follows readily that $\|f\|_{s, t} \leqslant(1+K) \gamma^{\prime}=\gamma$ for all $(s, t)$ satisfying $0 \leqslant s \leqslant t \leqslant b$. Since each $f$ vanishes outside $(0, b)$, condition (1) implies $\|f\|_{s, t} \leqslant \gamma$ for all finite $s, t, s \leqslant t$. If $b \leqslant \alpha$, the same result obviously holds. It is then immediate that $M$ is uniformly bounded over all $A_{s, t}$.

We show next that $M$ is totally bounded in each $A_{s, t}$. Since $L_{t} M=M$, it is sufficient by Lemma 4 to show that $M$ is totally bounded in $A_{0, b}$. Consider a sequence $\left\{f_{n}\right\}, f_{n} \in M$. Each $f_{n}=L_{\tau_{n}} f_{n}^{\prime}$ with $f_{n}^{\prime} \in M$. If there are infinitely many $\tau_{n}$ with $\left|\tau_{n}\right|>b$ the corresponding $f_{n}$ trivially form a Cauchy subsequence in $A_{0, b}$; in fact each $f_{n}$ in this subsequence has $\left\|f_{n}\right\|_{0, b}=0$. If there are only finitely many $\tau_{n}$ with $\left|\tau_{n}\right|>b$ then there is necessarily a subsequence $\left\{\tau_{1 n}\right\}$ with $\tau_{1 n} \rightarrow \tau$ in $[-b, b]$. There is then a subsequence $\left\{f_{2 n}\right\}$ with $\left\{\tau_{2 n}\right\}$ a subsequence of $\left\{\tau_{1 n}\right\}$ such that $\left\{f_{2 n}^{\prime}\right\}$ is Cauchy in $A_{0, b+\tau}$ if $\tau<0$ or in $A_{\tau, b}$ if $\tau<0$. Let it be understood that for 
what follows $n, m$ belong to the second subsequence of the integers. Then

$$
\begin{aligned}
\left\|f_{n}-f_{m}\right\|_{0, b}= & \left\|L_{\tau_{n}} f_{n}^{\prime}-L_{\tau_{m}} f_{m}^{\prime}\right\|_{0, b} \leqslant\left\|L_{\tau_{n}-\tau} f_{n}^{\prime}-f_{n}^{\prime}\right\|_{\tau, b+\tau} \\
& +\left\|L_{\tau_{m}-\tau} f_{m}^{\prime}-f_{m}^{\prime}\right\|_{\tau, b+\tau}+\left\|f_{n}^{\prime}-f_{m}^{\prime}\right\|_{\tau, b+\tau} .
\end{aligned}
$$

where $-b \leqslant \tau \leqslant b$. We recall that each $f_{n}^{\prime}$ vanishes outside $(0, b)$. Thus the third term is equal to $\left\|f_{n}^{\prime}-f_{m}^{\prime}\right\|_{0, b+\tau}$ if $\tau \leqslant 0$ and is dominated by $\left\|f_{n}^{\prime}-f_{m}^{\prime}\right\|_{\tau, b}$ if $\tau>0$. In either event, there is $N_{1}$ such that the third term does not exceed $\epsilon>0$ for $n, m \geqslant N_{1}$.

Let $N_{2}$ be such that $\left|\tau_{n}-\tau\right| \leqslant \alpha / 2$ for $n \geqslant N_{2}$. Then the first term is equal to $\left\|L_{\tau_{n}-\tau} f_{n}^{\prime}-f_{m}^{\prime}\right\|_{-\alpha / 2, b+\tau}$ if $\tau \leqslant 0$ and is dominated by $\left\|L_{\tau_{n}-\tau} f_{n}^{\prime}-f_{n}^{\prime}\right\|_{\tau_{i} b+\alpha / 2}$ if $\tau>0$. It follows from the hypotheses that $M_{0}$ is totally bounded in the spaces $A_{-\alpha, b+\tau-\alpha / 2}$ and $A_{b+\tau-\alpha / 2, b+\tau+\alpha / 2}$, for $-b \leqslant \tau \leqslant 0$, and in the spaces $A_{\tau-\alpha / 2, b}, A_{b, b+\alpha}$ for $0 \leqslant \tau \leqslant b$. Then for $m, n \geqslant N_{3} \geqslant N_{2}$ the first and second terms of (5) do not exceed $\epsilon$ by Lemma 6, uniformly in $f_{n}^{\prime}$. Thus for $m, n \geqslant \max \left(N_{1}, N_{3}\right)$, $\left\|f_{n}-f_{m}\right\|_{0, b} \leqslant 3 \epsilon$, so $\left\{f_{n}\right\}$ is Cauchy in $A_{0, b}$.

From Lemma 5 , it follows that $M$ is totally bounded in each $A_{t}$, and from Proposition 3 that $M$ is shift-continuous.

If $C$ has property $(S)$ one can take $M_{0}$ to be a totally bounded subset of $[a, a+\alpha]$, then $M_{0}$ is totally bounded in each $A_{s, t}, a \leqslant s \leqslant t \leqslant a+\alpha$.

Remark 4. It is easily seen that no $A^{t}$ can contain a totally bounded shift-invariant subset which contains a function not equivalent to zero and with support in a finite interval.

This fact plus the conclusion of Proposition 4 suggest that $A^{t}$ spaces are not satisfactory as input spaces-at least they cannot meet all the criteria stated in the Introduction. Thus any irregular family of spaces $\left\{A_{t}\right\}$ is not suitable as a family of input spaces, and in particular no $L_{p}$-space, $1 \leqslant p \leqslant \infty$, is suitable.

On the other hand, any family $\left\{A_{s, t}\right\}$ that is shift-continuous, tapered and has property $(S)$ will yield a suitable family $\left\{A_{t}\right\}$ by the preceding Proposition. Although the requirement that the family $\left\{A_{s, t}\right\}$ be tapered has not been shown to be necessary for the conclusion of Proposition 5, the preceding results give some evidence that something like this property is needed for the kind of system structure desired. Indeed, the property of being irregular can be interpreted as being very strongly non-tapered. Some further discussion of what tapering seems to mean is given in the next section.

The question as to whether the spaces $\left\{A_{t}\right\}$ are complete or not is largely irrelevant for our immediate purposes. Completeness is only needed for construction of examples involving limit processes, as in Proposition 3.

One can stipulate conditions on the class of functions $C$ so that if the spaces $A_{s, t}$ are complete, then $A_{t}$ will be complete, but this is not done here.

\section{System Trajectories}

Let $\left\{A_{s, t}\right\}$ be a fitted family of normed linear spaces with bounding space $A$. $U$ is a subset of $A$ with the properties: (i) $L_{t} U=U$; (ii) $U$ is totally bounded in $A_{0}$; (iii) $\left\|L_{h} u-u\right\|_{0} \rightarrow 0$ as $h \rightarrow 0$ for all $u \in U$. Sufficient conditions on $\left\{A_{s, t}\right\}$ for the 
existence of such a metric subspace $U$ are given in Proposition 5. Hypotheses (i) and (ii) imply that $U$ is totally bounded in each $A_{t}$ and each $A_{s, t}$ and is bounded in $A$; (i) and (iii) imply shift continuity with respect to any $\|\cdot\|_{s, t}$ or $\|\cdot\|_{t} . U$ is the input space for a system $(U, Y, F)$. We denote by $U_{t}$ the metric subspace of $A_{t}$ defined by regarding $U$ as a subset of $A_{t}$; thus (see preceding section) an element $u^{\prime} \in U_{t}$ can be regarded as an equivalence class of elements in $U$, where $u_{1} \sim u_{2}$ means $\left\|u_{1}-u_{2}\right\|_{t}=0$, or alternatively as an equivalence class of functions in $C$, where again $f_{1} \sim f_{2}$ means $\left\|f_{1}-f_{2}\right\|_{t}=0$.

Let $\left\{B_{s, t}\right\}$ be a fitted family of normed linear spaces with bounding space $B$ and with the property that $\left\|L_{h} y-y\right\|_{0} \rightarrow 0$ as $h \rightarrow 0$ for all $y \in B$. Henceforth, to avoid confusion, we use the notations $\|\cdot \cdot\|\left\|_{s, t},\right\| \cdot \cdot\|\|_{t},\|\mid \cdot\|$ for the norms in $B_{s, t}, B_{t}, B$, respectively. $B$ is the output space for $(U, Y, F)$. We also use the notation $Y_{t}=B_{t}$.

$F$ is a map from $U$ into $Y=B$ with the properties: (a) if $u_{1}, u_{2} \in U$ satisfy $\left\|u_{1}-u_{2}\right\|_{t}=0$ then $\left\|F\left(u_{1}\right)-F\left(u_{2}\right)\right\|_{t}=0$; thus $F$ induces a map $\tilde{F}_{t}$ from $U_{t}$ into $Y_{t}=B_{t}$; (b) $\tilde{F}_{t}$ is uniformly continuous and bounded for each $t$. We refer to $(U, Y, F)$ as the global system and $\left(U_{t}, Y_{t}, \tilde{F}_{t}\right)$ as a truncated system.

The condition (a) on $F$ forces $F$ to be causal, but it also imposes a further restriction. In general it is possible that two distinct elements $u_{1}, u_{2} \in U$ can correspond to the same element of $A_{t}$ and also to the same element of every $A_{s}$ for $s \geqslant t$. Heuristically speaking, $u_{1}$ and $u_{2}$ are distinct in $U$ because they differ in the remote past, but they do not differ in the future. An arbitrary causal map from $U$ into $B$ could carry $u_{1}$ and $u_{2}$ into different image points in $B_{t}$ and thus not even generate a map from $U_{t}$. Thus, condition (a) is part of the requirement that $F$ not be too sensitive to the remote past. The continuity condition (b) strengthens this requirement.

If $F$ has bounded finite memory this subtlety need not occur. In [1], for example the spaces are set up so that if a causal $F$ with bounded memory is continuous and bounded from $U$ into $Y, \tilde{F}_{t}$ is automatically well-defined and is also continuous and bounded.

The map $F_{t}: U_{0} \rightarrow Y_{0}$ is defined by*

$$
F_{t}(u)=L_{t} \tilde{F}_{t}\left(R_{t} u\right), u \in U_{0} .
$$

Each $F_{t} \in \mathscr{B}\left(U_{0}, Y_{0}\right)$, the Banach space of bounded continuous maps from $U_{0}$ into $Y_{0}$ with norm: $\left\|F_{t}\right\|=\sup _{u}\left\|F_{t}(u)\right\|_{0}, u \in U_{0}$. As $t$ runs through the real numbers, the mapping $t \rightarrow F_{t}$ describes a trajectory in $\mathscr{B}\left(U_{0}, Y_{0}\right)$.

Proposition 6. With $\left(U_{0}, Y_{0}, F_{t}\right)$ as specified, the trajectory $t \rightarrow F_{t}$ is continuous.

Proof.

$$
\begin{aligned}
\left\|F_{t}-F_{t+h}\right\| & =\sup _{u}\left\|\left(F_{t}-F_{t+h}\right) u\right\|_{0} \\
& =\sup _{u}\left\|L_{t} \tilde{F}_{t} R_{t} u-L_{t+h} \tilde{F}_{t+h} R_{t+h} u\right\|_{0}, \quad u \in U_{0} .
\end{aligned}
$$

*The notation $R_{t}=L_{-t}$ is used here. 
We note first that the supremum in the above expression can be regarded as being over all $u \in U$, since each element of $U$ is a member of an equivalence class defining an element in $U_{0}$. Second, by the causality of $F$ and the definition of $\tilde{F}_{t}$,

$$
\left\|L_{t} \tilde{F}_{t} R_{t} u-L_{t} F R_{t} u\right\|_{0}=0
$$

for all $t$ and all $u \in U$. Thus we can write

$$
\left\|F_{t}-F_{t+h}\right\|=\sup _{u}\left\|L_{t} F R_{t} u-L_{t+h} F R_{t+h} \dot{u}\right\|_{0}, u \in U .
$$

Then,

$$
\left\|F_{t}-F_{t+h}\right\| \leqslant \mathrm{I}+\mathrm{II}
$$

where

$$
\begin{aligned}
& \mathrm{I}=\sup _{u}\left\|L_{t} F R_{t} u-L_{t+h} F R_{t} u\right\| \|_{0} \\
& \mathrm{II}=\sup _{u}\left\|L_{t+h} F R_{t} u-L_{t+h} F R_{t+h} u\right\|_{0} .
\end{aligned}
$$

Since $U$ is shift-invariant,

$$
\mathrm{I}=\sup _{u}\|\| L_{t} F u-L_{t+h} F u\left\|_{0}=\sup _{u}\right\| F u-L_{h} F u\|\|_{t} .
$$

$F(U)$ is totally bounded when regarded as a subset of any $B_{s}$ since $U$ is totally bounded in $A_{s}$ and $\tilde{F}_{s}$ is uniformly continuous. Hence by uniform shift-continuity (Lemma 6 ) $\mathrm{I} \rightarrow 0$ as $h \rightarrow 0$.

$$
\begin{aligned}
\mathrm{II}= & \sup _{u}\left\|F R_{t} u-F R_{t+h} u\right\|_{t+h} \leqslant \sup _{u}\left\|F R_{t} u-F R_{t+h} u\right\| \|_{t-\alpha / 2} \\
& +K \sup _{u}\left\|F R_{t} u-F R_{t+h} u\right\|_{t+\alpha / 2}
\end{aligned}
$$

for $|h| \leqslant \alpha / 2$. Consider the first of the two terms bounding II. Again by uniform shift-continuity

$$
\left\|R_{t} u-R_{t+h} u\right\|_{t-\alpha / 2}=\left\|u-R_{h} u\right\|_{-\alpha / 2} \rightarrow 0
$$

as $h \rightarrow 0$, uniformly for $u \in U$. The uniform continuity of $\tilde{F}_{t-\alpha / 2}$ then gives

$$
\sup _{u}\left\|F R_{t} u-F R_{t+h} u\right\|_{t-\alpha / 2} \rightarrow 0 .
$$

The second term bounding II is treated in exactly the same fashion.

It is easy to construct examples of systems for which a truncated system trajectory is not continuous when various of the hypotheses of the preceding Proposition are not satisfied. We give one simple example for which $U$ and $Y$ are shift-invariant and shift-continuous, and each $\tilde{F}_{t}$ is continuous and bounded. 
$U$ is totally bounded in each $A_{s, t}$ for finite $s, t$, but fails to meet the conditions of Proposition 6 in that it is not totally bounded in $A_{t}$.

We take $A_{s, t}$ to be the Lebesgue space $L_{1}(s, t)$, with real-valued functions, so that $A_{t}$ is $L_{\mathrm{l}}(-\infty, t)$. $U$ consists of the function $u_{0}$ and all its translates, where

$$
\begin{aligned}
u_{0}(t) & =1,0<t \leqslant 1 / 2 \\
& =0 \text { otherwise }
\end{aligned}
$$

Clearly $U$ satisfies the conditions mentioned but is not totally bounded in $A_{0}$.

The $B_{s, t}$ are also $L_{1}$-spaces of real-valued functions. If $(t-s)<1, B_{s, t}$ is $L_{1}(s, t)$; if $(t-s) \geqslant 1, B_{s, t}$ is $L_{1}(t-1, t)$. Thus $Y_{t}=B_{t}$ is also $L_{1}(t-1, t)$ (see Example B). The input-output map $F$ is defined by

$$
[F(u)](t)=0,-1<t \leqslant-1 / 2,=\sum_{n=1}^{\infty} 2^{n} \int_{-n}^{-n+2^{-n}} u(s) d s,-1 / 2<t \leqslant 0
$$

for the interval $(-1,0]$. The defining equations are translated by $k$ for the interval $(-1+k, k], k$ an integer. The infinite sum is just a notational convenience, since for any $u \in U$ at most only one term of the sum is nonzero. Obviously $F$ is causal, also $[F(u)](t) \leqslant 1$ for all $u \in U$ and all $t$, so that

$$
\left\|\tilde{F}_{t}\right\|=\sup _{u}\|F(u)\|_{t}=1 / 2 .
$$

$\tilde{F}_{t}$ is continuous on $U_{t}$, although not uniformly so of course.

We now have

$$
\left\|F_{h}-F_{0}\right\|=\sup _{u}\left\|L_{h} F R_{h} u-F u\right\|_{0}>1 / 4 \text { (say) }
$$

for any $|h|<1 / 2$, since for an arbitrarily small $h$ some $u$ far enough to the left of the origin will yield $[F(u)](t)=0,-1 / 2<t \leqslant 0$ but $\left[F\left(R_{h} u\right)\right](t)=1,-1 / 2<t \leqslant 0$.

Remark 5. This example illustrates how difficulty can arise because the input-output map has "too much memory." If $F$ were defined as it is above except with a finite sum so as to have bounded memory, the trajectory would be continuous - or if the "divergence factors" $2^{n}$ were eliminated the trajectory would be continuous.

It is not claimed that the hypotheses of Proposition 6 cannot be weakened. However the theorem invokes. Only what seem to be natural hypotheses. It includes the trajectory continuity results in [1] and [2] and is applicable to a wide variety of normed linear space topologizations.

A theorem of the type of the above is of interest, to the author at least, for two reasons. First, a time-varying system that fails to have continuous truncated-system trajectories shows a fundamental type of instability. It suddenly changes its mode of operation. If one is devising a mathematical model of a real-life system, he should at least be careful not to introduce such possible instability artificially when there is no evidence such behavior can occur in the 
real system. If such behavior can occur, that is an interesting fact in itself. Second, the continuity of trajectories is one essential requirement for the evolution of the system to be determined by a continuous semigroup of linear operators; i.e. for the system to have the hyperlinear, hypertime-invariant property mentioned in the Introduction. The other requirement is that the trajectories "behave like dynamical system trajectories," i.e. do not cross themselves or each other, do not split, etc. We conclude by establishing conditions for such a semigroup. This is done in essentially the same fashion as for the special case in [2].

Let $U$ and $Y$ satisfy the conditions of Proposition 6 , and $\mathcal{H}$ be a set of maps $F: U \rightarrow Y$ each of which satisfies the conditions of Proposition 6, Note that the set $\mathscr{F}$ of all $F$ that satisfy these conditions is a linear space. Let $\mathscr{T}=V(\mathcal{H})$, the linear span of $\mathcal{H}$ in $\mathscr{F}$. The mapping $\pi_{t}$ defined by $\pi_{t} F=F_{t}$ is linear on $\mathscr{F}$, and we put $\mathscr{K}_{t}=\pi_{t}(\mathfrak{N}), t \in R$. $\mathscr{N}_{t}$ is a linear subset of the Banach space $\mathscr{B}\left(\mathscr{Q}_{0}, \mathscr{Y}_{0}\right)$; its closure in $\mathscr{B}\left(\mathscr{U}_{0}, \mathscr{Y}_{0}\right)$ is denoted $\overline{\mathscr{N}}_{t}$.

Now suppose the following condition is satisfied.

(SG) For $F, T \in \mathscr{N}$, if $\pi_{t} F=\pi_{s} G$ for some $t, s \geqslant 0$, then $\pi_{t+a} F=\pi_{s+a} G$ for all $a \geqslant 0$.

If $H \in \Re_{t}$, let

$$
\pi_{t}^{-1} H=\left\{F \in \mathscr{T}: \pi_{t} F=H\right\} .
$$

Define an operator $\theta(t, s) \mathfrak{M}_{t} \rightarrow \mathfrak{M}_{s}, 0 \leqslant t \leqslant s$ by

$$
\theta(t, s) H=\pi_{s}^{\circ} \pi_{t}^{-1} H
$$

$\theta(t, s)$ is well-defined and linear. Now suppose that $H \in \mathfrak{N}_{t}$ and also $H \in \mathfrak{N}_{t+a}$, $a>0$. Then $H=\pi_{t} F=\pi_{t+a} G$ for some $F, G \in \Re$. Thus, for $s>t$,

$$
\begin{aligned}
\theta(t, s) H & =\pi_{s} \circ \pi_{t}^{-1}\left(\pi_{t} F\right)=\pi_{s} F \\
\theta(t+a, s+a) H & =\pi_{s+a}{ }^{\circ} \pi_{t+a}^{-1}\left(\pi_{t+a} G\right)=\pi_{s+a} G .
\end{aligned}
$$

By condition $(S G), \pi_{s} F=\pi_{s+a} G$ so that

$$
\theta(t, s) H=\theta(t+a, s+a) H .
$$

Now define $\theta(\tau)$ by

$$
\theta(\tau) H=\theta(t, s) H, s=t+\tau, \tau \geqslant 0, t \geqslant 0,
$$

for all $H$ such that for some $t, H \in \mathfrak{M}_{t}$. The calculation just made shows that $\theta(\tau)$ is well-defined, because if more than one pair $(t, s)$ satisfy the conditions they all yield the same $\theta(t, s) H$. The domain of $\theta(\tau)$, for any $\tau \geqslant 0$, includes $\cup_{t \geqslant 0} \mathscr{R}_{t}$. This may be extended by linearity to include $\mathscr{R}_{+}=V\left(\cup_{t \geqslant 0} \mathfrak{R}_{t}\right)$. The family $\{\theta(\tau), \tau \geqslant 0\}$ is a one-parameter semigroup of linear transformations on $\vartheta_{+}$. If the $\theta(\tau)$ are bounded linear operators (this amounts, of course, to requiring the trajectories to vary continuously with respect to an initial point) 
they may be extended by continuity to $\overline{\mathscr{N}}_{+}$, the closure of $\mathscr{T}_{+}$in $\Re\left(U_{0}, X_{0}\right)$.

Then we have:

Proposition 7. Let $U$ and $X$ satisfy the conditions of Proposition 6. Let $\mathcal{H}$ be a class of maps $F: U \rightarrow Y$ that satisfy the conditions of Proposition 6, and let $\mathcal{H}$ also satisfy $(S G)$. Then if the $\theta(\tau)$ as defined above are bounded operators and are uniformly bounded in a neighborhood of the origin, $\{\theta(\tau), \tau \geqslant 0\}$ is a strongly continuous semigroup of linear operators on the Banach space $\overline{\mathfrak{\Re}}_{+}$.

Proof. There is nothing remaining to prove except that $\{\theta(\tau), \tau \geqslant 0\}$ is strongly continuous, but this follows immediately from the continuity of the trajectories (see [1]).

The semigroup $\theta(\tau)$ generates the truncated-system trajectories for all the systems in $\mathcal{H}$ and thus gives the prediction for future system behavior. As in any dynamical system theory, prediction is possible only because each "state" of the system is a point on a trajectory which is distinct from all other trajectories as time is increased. The role of state is taken here by the truncated system map $F_{t}$; the condtion $(S G)$ assures the proper trajectory characteristics. The fact that $\theta(\tau)$ is linear comes about, of course, because $(S G)$ is required to hold for the extension of $\pi_{t}$ to $V(\mathcal{H})$. Thus the existence of the semigroup $(\theta(\tau)$ depends on genuinely restrictive conditions, as must be expected. However, it is implicit in the results of Section 2 and Proposition 6 that getting the semigroup to be strongly continuous is pretty much a matter of choosing suitable spaces for the system model.

Some further results are obtained in [1] on the existence of the strongly continuous semigroup as the finite observation interval length is changed, and also some results on approximation of trajectories. One would expect analogues of these results to hold in the more general case treated here, but no effort is made here to investigate this.

\section{Appendix}

Proof of Proposition 4. We construct a function $h=\sum_{n=0}^{\infty} h_{n}$ which has the stated properties. The functions $h_{n}$ are defined by

$$
h_{n}=L_{\delta_{n}} f_{n}
$$

where the numbers $\delta_{n}$ and the functions $f_{n}$ are as specified below.

Let $\alpha>0, K \geqslant 1$ satisfy condition (5). By property $(S)$ there are functions $f_{n}, n=0,1, \ldots$, which satisfy the conditions:

$$
\begin{aligned}
& f_{n}(t)=0, t \notin I_{n} \subset\left(\frac{-\alpha}{n+1}, 0\right) \\
& \left\|f_{n}\right\|_{-\frac{\alpha}{n+1}, 0}=\left\|f_{n}\right\|_{0}=1 .
\end{aligned}
$$

The numbers $\delta_{n}>0$ are chosen large enough to satisfy the following conditions 
for all $g \in G(2+2 K, 0)$. This is possible because $\left\{A_{s, t}\right\}$ is tapered.

$$
\begin{aligned}
\delta_{1}: & \delta_{1}>2 \alpha, \\
& \|g\|_{0}-\|g\|_{-\delta_{1}, 0} \leqslant 2^{-1} \\
\delta_{2}: & \delta_{2}>\delta_{1}+2 \alpha \\
& \|g\|_{0}-\|g\|_{-\delta_{2}, 0} \leqslant 2^{-2} \\
& \|g\|_{-\delta_{1}}-\|g\|_{-\delta_{2},-\delta_{1}}<2^{-1} \\
\delta_{3}: & \delta_{3}>\delta_{2}+2 \alpha \\
& \|g\|_{0}-\|g\|_{-\delta_{3}, 0} \leqslant 2^{-3} \\
& \|g\|_{-\delta_{1}}-\|g\|_{-\delta_{3},-\delta_{1}} \leqslant 2^{-2} \\
& \|g\|_{-\delta_{2}}-\|g\|_{--\delta_{3},-\delta_{2}} \leqslant 2^{-1}
\end{aligned}
$$

etc.

Now, for $m \leqslant M<N$,

$$
\left\|\sum_{n=M}^{N} h_{n}\right\|_{-\delta_{m}} \leqslant \sum_{n=M}^{N}\left\|h_{n}\right\|_{-\delta_{m}}=\sum_{n=M}^{N}\left\|L_{\delta_{n}} f_{n}\right\|_{-\delta_{m}}
$$

But $\left\|L_{\delta_{n}} f_{n}\right\|_{t}=\left\|f_{n}\right\|_{t+\delta_{n}} \leqslant K$ for all $t$, hence $L_{\delta_{n}} f_{n} \in G(2+2 K, 0)$ and

$$
\left\|L_{\delta_{n}} f_{n}\right\|_{-\delta_{m}}-\left\|L_{\delta_{n}} f_{n}\right\|_{-\delta_{n},-\delta_{m}} \leqslant 2^{-(n-m)}
$$

Since

$$
\left\|L_{\delta_{n}} f_{n}\right\|_{-\delta_{n},-\delta_{m}}=0, \quad\left\|L_{\delta_{n}} f_{n}\right\|_{-\delta_{m}} \leqslant 2^{-(n-m)} .
$$

Thus,

$$
\left\|\sum_{n=M}^{N} h_{n}\right\|_{-\delta_{m}} \leqslant \sum_{n=M}^{N} 2^{-(n-m)}=\sum_{p=M-m}^{N-m} 2^{-p}
$$

which approaches zero as $N, M \rightarrow \infty$ with $m$ fixed. Since $A_{-\delta_{m}}$ is a Banach space $h$ exists as an element of $A_{-\delta_{m}}$ for each $m=1,2, \ldots$ It follows readily that $h$ exists as an element of each $A_{t}, t \leqslant 0$.

Furthermore,

$$
\|h\|_{-\delta_{m}} \leqslant \sum_{n=0}^{\infty}\left\|h_{n}\right\|_{-\delta_{m}} \leqslant \sum_{n=0}^{\infty} 2^{-n}=2
$$

for all $m$. Then, if $t \leqslant 0$ does not lie in the support of one of the $h_{n}$,

$$
\|h\|_{t} \leqslant\|h\|_{-\delta_{m}} \text { for some } m \text {. }
$$

If $t$ does lie in the support of one of the $h_{n}$ the standard argument involving 
condition (5) yields

$$
\|h\|_{t} \leqslant 2+2 K, \quad t \leqslant 0
$$

Hence, $h \in G(2+2 K, 0)$.

Now, for any $\tau$, satisfying $|\tau|<\alpha$,

$$
\left\|L_{\tau} h-h\right\|^{0}=\sup _{-\infty<t \leqslant 0}\left\|L_{\tau} h-h\right\|_{t} \geqslant\left\|L_{\tau} h-h\right\|_{t^{1}, t}
$$

for any $t^{1}<t \leqslant 0$. Choose $n$ large enough that $\frac{\alpha}{n+1}<\frac{\tau}{2}$, and take $\left[t^{1}, t\right]$ to be the support of $h_{n}$. $L_{\tau} h$ then vanishes on $\left[t^{1}, t\right]$, so

$$
\left\|L_{\tau} h-h\right\|_{t^{1}, t}=\|h\|_{t^{1}, t}=\left\|L_{\delta_{n}} f_{n}\right\|_{t^{1}, t}=\left\|f_{n}\right\|_{-\frac{\alpha}{n+1}, 0}=1 .
$$

Thus $L_{\tau} h$ does not converge to $h$.

\section{References}

1. W. L. Root, On the modelling of systems for identification. Part 2. Time-varying systems, SIAM J. Control, 13, 4, 945-974 (1975).

2. W. L. Root, A semigroup representation of linear classes of causal, time-varying, nonlinear systems, Proc. of Ninth Annual Asilomar Conf. on Circuits, Systems, and Computers, 238-241 (1975).

Received July 5, 1978 